\title{
Capacidad de inclusión de las fintech en la Bolsa de Valores de Colombia
}

\author{
Fundación Universitaria de la Cámara de Comerc \\ Erika Salazar-Marín ${ }^{1}$
otá - Uniempresarial \\ erikasalazar.m14@gmail.com
}

\section{DOI: https://doi.org/10.21158/23227230.v9.n0.2019.2563}

Cómo citar este artículo: Salazar-Marín, E. (2019). Capacidad de inclusión de las fintech en la Bolsa de Valores de Colombia. Revista Ploutos, (Páginas). DOI: https://doi.org/10.21158/23227230.v9.n0.2019.2563

Fecha de recepción: 29 de septiembre de 2019

Fecha de aprobación: 11 de octubre de 2019

\section{Resumen}

Las fintech han sido una parte fundamental en el crecimiento económico. Debido a sus avances tecnológicos han ayudado a múltiples empresas a realizar todo tipo de transacciones y han facilitado la vida de millones de personas. Al realizar esta investigación, el objetivo fue analizar la evolución histórica de las fintech y su impacto al momento de invertir en el mercado colombiano, en busca de ser reconocidas en el mercado financiero, de innovar tecnologicamente y generar confiabilidad de forma más efectiva. Para esta investigación fueron utilizados como datos de análisis gráficos, datos históricos de precios y estadísticas de empresas en otros países, como Alibaba, por ejemplo. Si bien al concluir la investigación los resultados no fueron los esperados, se pudo establecer que a futuro las fintech pueden ser una opción de inversión en el mercado colombiano, debido al reconocimiento que este tiene en el sector financiero y sus múltiples desarrollos tecnológicos, ya que en el mundo existe una tendencia a usar medios digitales cada vez más eficientes que ayudan a hacer diversas actividades de manera más sencilla.

Palabras clave: fintech, mercado financiero; Bolsa de Valores de Colombia; cotización en bolsa de valores; inversión; acciones fintech.

\section{Ability to include fintech in the Colombian Stock Exchange}

\begin{abstract}
The fintech companies have been a fundamental part of economic growth. Due to their technological advances, they have helped many companies conduct all kinds of transactions, facilitating the lives of millions of people. In carrying out this research, the objective was to analyze the historical evolution of the fintech industry and its impact when investing in the Colombian market, with the expectation of becoming well known in the financial market, innovating in technology, and generating reliability in a more effective way. This research used historical pricing data and statistics of companies in other countries, such as Alibaba, as graphical analysis data. Although at the end of the investigation the results were not as expected, it was possible to establish that in the future, the fintech industry can be considered an option for investment in the Colombian market, due to the recognition that it has in the financial sector and its multiple technological developments, as there is a worldwide tendency to use increasingly efficient digital media that help develop different activities in an easier manner.
\end{abstract}

Keywords: fintech, financial market; Colombia Stock Exchange; stock market listing; investment; fintech shares.

\footnotetext{
${ }^{1}$ Estudiante del programa de Finanzas y comercio exterior en la Fundación Universitaria de la Cámara de Comercio de Bogotá - Uniempresarial. ORCID: https://orcid.org/0000-0001-9097-2070
} 


\section{Capacidade de incluir as fintech na Bolsa de Valores da Colômbia}

\section{Resumo}

As empresas fintech têm sido uma parte fundamental do crescimento econômico. Devido a seus avanços tecnológicos, elas ajudaram várias empresas a realizar todos os tipos de transações e facilitaram a vida de milhões de pessoas. Na realização desta pesquisa, o objetivo foi analisar a evolução histórica das fintech e seu impacto ao investir no mercado colombiano, buscando ser reconhecida no mercado financeiro, inovar tecnologicamente $e$ gerar confiabilidade com mais eficácia. Para esta pesquisa, foram utilizados como dados de análise gráfica, dados históricos de preços e estatísticas de empresas de outros países, como o Alibaba, por exemplo. Embora no final da investigação os resultados não tenham sido os esperados, foi estabelecido que, no futuro, a fintech poderá ser uma opção de investimento no mercado colombiano, devido ao reconhecimento que possui no setor financeiro e seus múltiplos desenvolvimentos tecnológicos, uma vez que existe uma tendência no mundo de usar mídias digitais cada vez mais eficientes que ajudam a realizar várias atividades com mais facilidade.

Palavras-chave: fintech, mercado financeiro; Bolsa de Valores colombiana; cotação na bolsa de valores; investimento; ações da fintech.

\section{De la possibilité d'introduire les fintech à la Bourse colombienne}

\section{Résumé}

Les fintech ont été un élément fondamental de la croissance économique. Grâce à leurs avancées technologiques, elles ont pu aider nombre d'entreprises à effectuer

toutes sortes de transactions et ont facilité la vie de millions de personnes. Cette investigation a pour but d'analyser l'évolution historique des fintech et leur impact positif lors d'investissements sur le marché colombien en terme d'innovations technologiques et de fiabilité. Lors de cette investigation nous avons utilisé des données d'analyse graphique, des données historiques sur les prix et des statistiques d'entreprises de pays tiers, comme l'entreprise Alibaba. Bien que les résultats de cette investigation aient été différents de ceux attendus au départ, il est possible d'établir qu'à l'avenir les fintech pourraient être une option d'investissement sur le marché financier colombien grace à la reconnaissance du secteur et de ses multiples avancées technologiques. En effet, la tendance actuelle est l'utilisation croissante d'entreprises numériques plus efficicientes permettant de réaliser plus aisément nombre d'opérations financières.

Mots-clés: fintech, marché financier; bourse colombienne; cotation en bourse; investissement; actions fintech

\section{Introducción}

Aprender sobre finanzas en el siglo XXI se ha hecho necesario debido a los grandes cambios. Era difícil pensar en la forma en que se ejecutarían pagos por medio de internet, ya que parecía inseguro y no se contaba con los suficientes parámetros para realizarlos. Ahora es un hecho. En las grandes potencias se han implementado prácticas como, por ejemplo, compras en tiendas 100 \% en línea, sin salir de casa y con dinero plástico, realizar retiros sin tarjetas o generar prestamos de efectivo desde casa, entre otros. 
Se puede decir que esto ocurrió en parte gracias a las fintech. Estas startups ${ }^{2}$, con sus ideas de negocio, han logrado crear plataformas online que han facilitado la vida de muchas personas en el mundo. Los usuarios dejaron el miedo a realizar transacciones o consultas virtuales y empezaron a utilizar estos servicios con más frecuencia, lo que le dio un crecimiento al mercado de forma considerable.

En poco tiempo, las empresas fintech en Colombia han impactado el mercado y han tenido una gran acogida, aunque algunos de sus nombres no sean reconocidos. Es un hecho que en un futuro no muy lejano se va a depender más de medios electrónicos, páginas web o aplicaciones celulares como medio de acceso a los servicios financieros, lo que hace necesario profundizar en las variables que puedan incidir en el momento de invertir en la Bolsa de Valores de Colombia o BVC, el cual no es un mercado de valores muy desarrollado, pero del que se pueden sacar provecho para crear oportunidades de crecimiento.

Al comparar una fintech que podría cotizar en la BVC con una empresa que cotiza en la bolsa de Nueva York es posible determinar que presentaría un comportamiento favorable en futuras inversiones.

Ahora bien, en este sentido, el objetivo general de la investigación es:

- Analizar la evolución histórica de las fintech y su impacto en el mercado de valores colombiano, por medio de gráficos y estadísticas.

En cuanto a los objetivos específicos, se tienen:

- Identificar los orígenes de las fintech y su evolución.

- Analizar los requerimientos mínimos para que las empresas fintech inviertan en la BVC.

\footnotetext{
2 Podría definirse una startup como una empresa emergente, normalmente con un alto componente tecnológico, con grandes posibilidades de crecimiento y que, por lo general, respalda una idea innovadora que sobresale de la línea general del mercado (Cañete, 2018).
} 
- Determinar el comportamiento de las acciones de Nueva York con el supuesto de inversión en Colombia, a través de precios históricos.

\section{Marco teórico}

\section{1 ¿Qué es?}

A fin de tener una idea de lo que son las fintech y cuál ha sido su trayectoria desde que se implementaron en Colombia, es necesario primero definir el término:

Las fintech - finance + technology - son empresas innovadoras que están emergiendo en los últimos años y que ofrecen nuevas soluciones financieras con el soporte de las nuevas tecnologías [...]. Ofrecen soluciones tanto para personas físicas como para empresas, y son auténticas especialistas en áreas concretas de los servicios financieros. (Molina, 2016)

Por otra parte, es importante resaltar que las fintech en Colombia toman fuerza cuando se funda la Asociación Colombia Fintech como entidad, cuyo propósito es incentivar y dar a conocer estas empresas emergentes. La Asociación Colombia Fintech se define de la siguiente manera: «Somos la asociación de empresas Fintech de Colombia, nacida en diciembre de 2016, con el fin común de crear un ecosistema dinámico de talento, capital y adopción para el desarrollo de los negocios fintech en este país» (Colombia Fintech, 2018).

De acuerdo con lo anterior, las fintech en Colombia han aportado a las entidades el libre desempeño y la formación a nivel tecnológico, dando apertura a nuevos procesos. La innovación de las fintech ha transformado la idea que tenía el país con respecto a la digitalización, tanto así que ya se han establecido entidades estatales con el fin de incentivar el emprendimiento por medio de la tecnología.

\subsection{Antecedentes}

En el mundo se ha analizado de varias formas la implementación de métodos dirigidos a mejorar la comunicación en el nivel financiero de las entidades. En el siglo XXI se prioriza el avance digital en el área financiera, en procesos cotidianos, así como la disminución de los tiempos de ejecución. 
En la tabla 1 se expone una breve línea de tiempo que permite reconocer la evolución de las fintech a nivel mundial.

Tabla 1. Historia de las fintech en el mundo

\begin{tabular}{|l|l|l|l|}
\hline $\mathbf{1 9 9 5}$ & $\mathbf{1 9 9 8}$ & $\mathbf{1 9 9 9}$ & $\mathbf{2 0 0 4}$ \\
\hline $\begin{array}{l}\text { Servicios bancarios a través } \\
\text { de la implementación de la } \\
\text { World Wide Web - }\end{array}$ & $\begin{array}{l}\text { Nace Cofinity, que después } \\
\text { sería PayPal. Su objetivo } \\
\text { era implementar un } \\
\text { sistema de pago con } \\
\text { tarjeta bancaria. }\end{array}$ & $\begin{array}{l}\text { Se funda Alibaba Group } \\
\text { Holding -China-. }\end{array}$ & Se crea Alipay-Alibaba. \\
\hline 2005 & 2009 & 2011 & 2014 \\
\hline $\begin{array}{l}\text { En Inglaterra se funda por } \\
\text { primera vez un banco sin } \\
\text { sucursal. }\end{array}$ & $\begin{array}{l}\text { Creación de la plataforma } \\
\text { Kickstarter- New York, se } \\
\text { conoce el término de } \\
\text { bitcoin. }\end{array}$ & $\begin{array}{l}\text { Nace el servicio de } \\
\text { transferwise - } \\
\text { transferencias-. }\end{array}$ & $\begin{array}{l}\text { Se crea Ant Financial } \\
\text { Services Group. Empresa } \\
\text { con seis de negocios. }\end{array}$ \\
\hline
\end{tabular}

Fuente. Camara de Comercio de Bogotá, 2018.

En la figura 1 se evidencia el impacto que ha causado el fenómeno de las fintech, el cual lleva un poco más de dos años en Colombia, además de los logros que desde su origen han transformado la metodología y la idea de una Colombia más digitalizada.

Figura 1. Colombia Fintech 2017

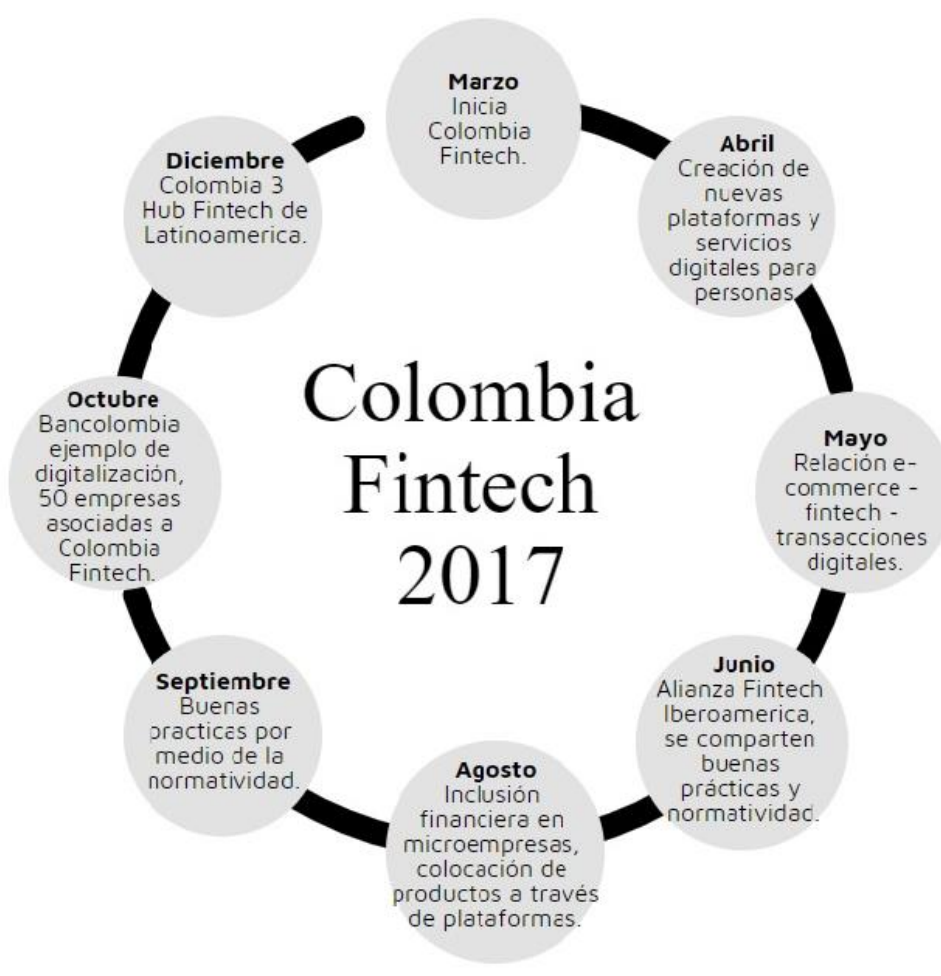

Fuente. Diagrama creado en Venngage, 2019, con datos de Colombia Fintech, s. f. 
En su primer año, las fintech crearon alianzas con varias entidades, lo que permitió cambios en los procesos a nivel bancario o financiero y que en la actualidad han impactado la vida cotidiana. Colombia, por medio de la asociación fintech, ha logrado llegar a lugares recónditos que muchas veces las entidades financieras no pueden alcanzar y esto ha incentivado la inclusión financiera en el país.

Figura 2. Colombia Fintech 2018-2019

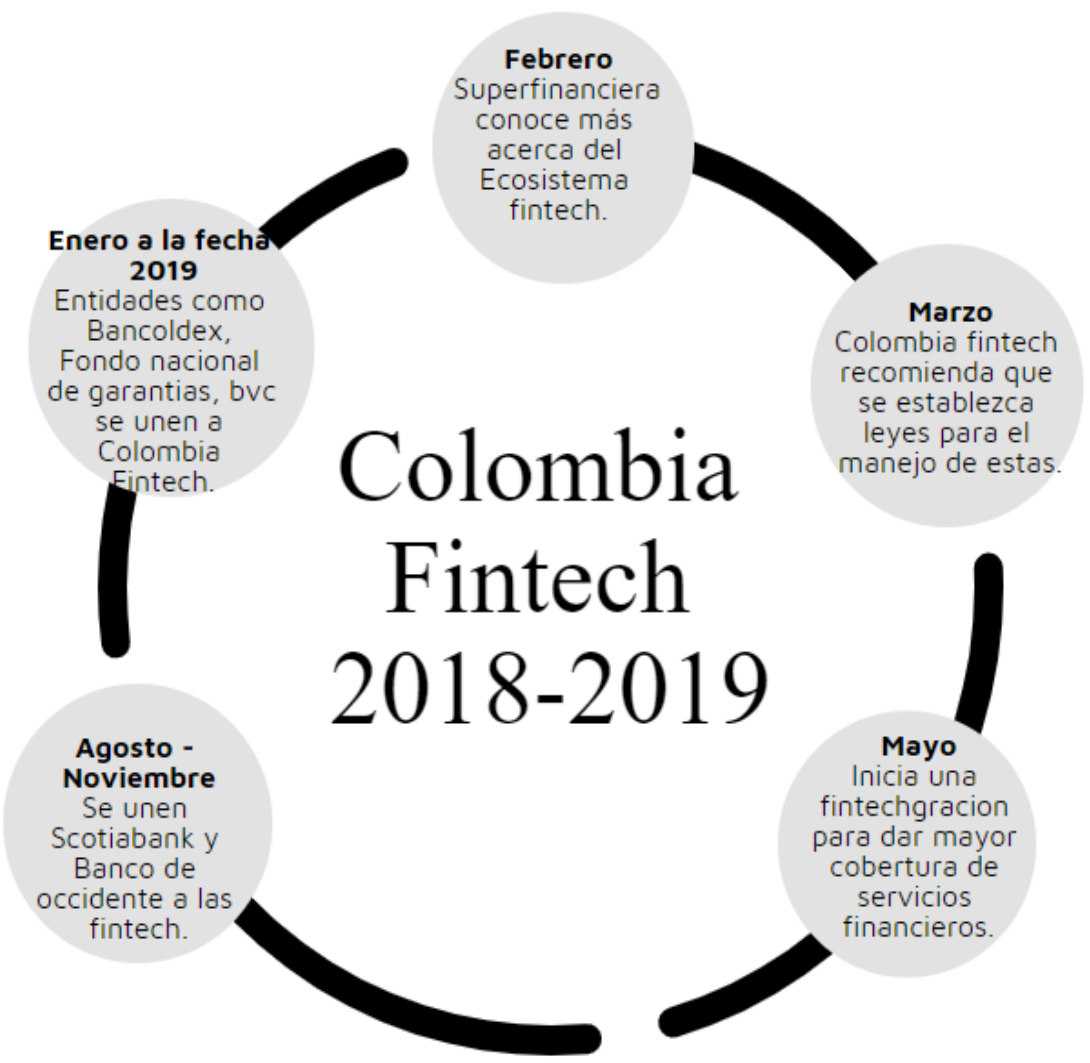

Fuente. Diagrama creado en Venngage, 2019, con datos de Colombia Fintech, s. f.

Es indispensable la creación de una norma más específica que establezca las pautas del ecosistema fintech y su funcionamiento, ya que no es suficiente con el Decreto 1357 de 2018, «por el cual se modifica el Decreto 2555 de 2010 en lo relacionado con la actividad de financiación colaborativa» (Libro 41, parte 2), el propio Decreto 2555 de 2010, u otras normativas como, por ejemplo, la Ley 1735 de 2014 y el Decreto 1491 de 2015. En ejercicio de sus facultades constitucionales y legales, en especial las conferidas por los numerales 11 
y 25 del artículo 189 de la Constitución Política, el literal n) del artículo 46 y los literales a) y r) del numeral 1 del artículo 48 del Estatuto Orgánico del Sistema Financiero, así como los artículos $1^{\circ}$ y $2^{\circ}$ de la Ley 1735 de 2014 , conviene subrayar que el Estado ha creado entidades que han apalancado el emprendimiento en este rubro, tal como lo realizó la Superintendencia Financiera a través del grupo de trabajo InnovaSFC (innovación financiera y tecnológica), el cual cuenta con la supervisión de esta entidad regulatoria (Asobancaria, 2018).

\subsection{Estructura de cotización de BVC}

De acuerdo con lo establecido por la BVC (2018), para que una empresa u otra entidad pueda cotizar en la bolsa, debe cumplir con los siguientes requisitos:

- Por lo menos el $10 \%$ del total de las acciones en circulación del emisor está en cabeza de personas distintas a aquellas que conforman un mismo beneficiario real. Este requisito evita el monopolio, ya que las acciones no pueden pertenecer a un solo propietario. Si fuera así no permitiría una competencia legal entre las empresas.

- El emisor tiene como mínimo 100 accionistas. Es decir, generar mayor uso de los recursos e ingresos dentro de la compañía.

- El emisor cuenta con un patrimonio de al menos COP 7000000 000. Esto es, una empresa o entidad sólida que pueda cumplir con sus obligaciones financieras en caso de alguna pérdida o de un incidente.

- El emisor o su controlante han ejercido su objeto social principal durante los tres años anteriores a la fecha de solicitud de inscripción. Esto quiere decir que la actividad que realiza la entidad debe estar acorde con sus ingresos operacionales, de manera que no presente algún inconveniente legal que pueda afectar su credibilidad y confianza.

- El emisor ha generado utilidades operacionales en alguna de las tres vigencias anuales anteriores al año en que se solicita la inscripción. La empresa debe tener ganancias en los últimos tres años previos a la inscripción.

- El emisor cuenta con una página web en la cual se encuentre a disposición del público la información que establece la BVC mediante su Circular Única. 
- El emisor se compromete a presentar un informe anual de gobierno corporativo que contenga la información que requiere la BVC mediante su Circular Única. Es decir, enviar informes periódicos de la situación financiera de la empresa.

\section{Marco metodológico}

En el marco metodológico se realiza un estimativo que puede dar como resultado un valor porcentual o relativo por medio de gráficos y estadísticas históricas que se analizan financieramente, los cuales ayuden a observar el comportamiento de las acciones de dos empresas que invierten en la Bolsa de Valores de New York - Paypal y Alibaba-, a fin de estar en capacidad de contrastarlas con las del mercado colombiano, lo que permite identificar el impacto del país o de la BVC.

En este orden de ideas, a continuación se profundiza un poco sobre las dos empresas. Primero, sobre PayPal, y luego Alibaba, la cual maneja una de las empresas fintech más importantes del mundo: Ant Financial.

PayPal es una de las pioneras fintech. Fundada en 1998 por Ken Howey y Max Levchin, se llamó inicialmente Cofinity. Más adelante se asocian Elon Musk, Peter Thiel y Luke Nosek, lo cual llevó a que se cambiara el nombre de la organización a PayPal. En la actualidad, es una de las empresas más importantes de servicios financieros en el mundo. Sus avances han sido extraordinarios, ya que gracias a esta empresa ha sido posible prestar servicios financieros alrededor del mundo con dinero plástico por medio de tarjetas de crédito y débito.

En el 2002, eBay compra la plataforma PayPal por unos 1500 dólares, en razón al gran ascenso de la compañía, ya que logró que un millón de usuarios utilizaran la plataforma. Después de esto, PayPal adquirió Zong, que manejaba en ese momento transacciones en dispositivos móviles, lo que la ayudó a destacarse como uno de los mejores canales de pagos del mundo. Lo que se conoce en la actualidad es que PayPal tiene 152 millones de usuarios activos con transacciones que ascienden a 27000 millones de dólares (Barrera, 2019). 
En el 2015 PayPal empieza a invertir en la Bolsa de Valores de New York e inicia con una operación bajista en lo que concierne a su apertura en el mercado. En el transcurrir de los años 2016 y 2017 la acción empezó a tomar fuerza y a tornar en una posición alcista. En aquellos años se disputaba la presidencia de Estados Unidos (EE. UU.). Los candidatos eran Hillary Clinton por el Partido Demócrata y Donald Trump por el Partido Republicano, ambos con diferentes ideologías frente a cómo tomar el mandato de una de las potencias más grandes; uno con beneficios al pueblo y otro con ideas económicas que pretendía beneficiar más al estadounidense y reformar las leyes de los inmigrantes en EE. UU. (Avendaño, 2016). Al posicionarse Donald J. Trump como presidente de los EE. UU., el 20 de enero del 2017, sus nuevas políticas causaron cierto revuelo a nivel internacional por la protección que se iba a brindar a sus ciudadanos (Los momentos destacados, 2017).

Figura 3. Comportamiento acción PayPal

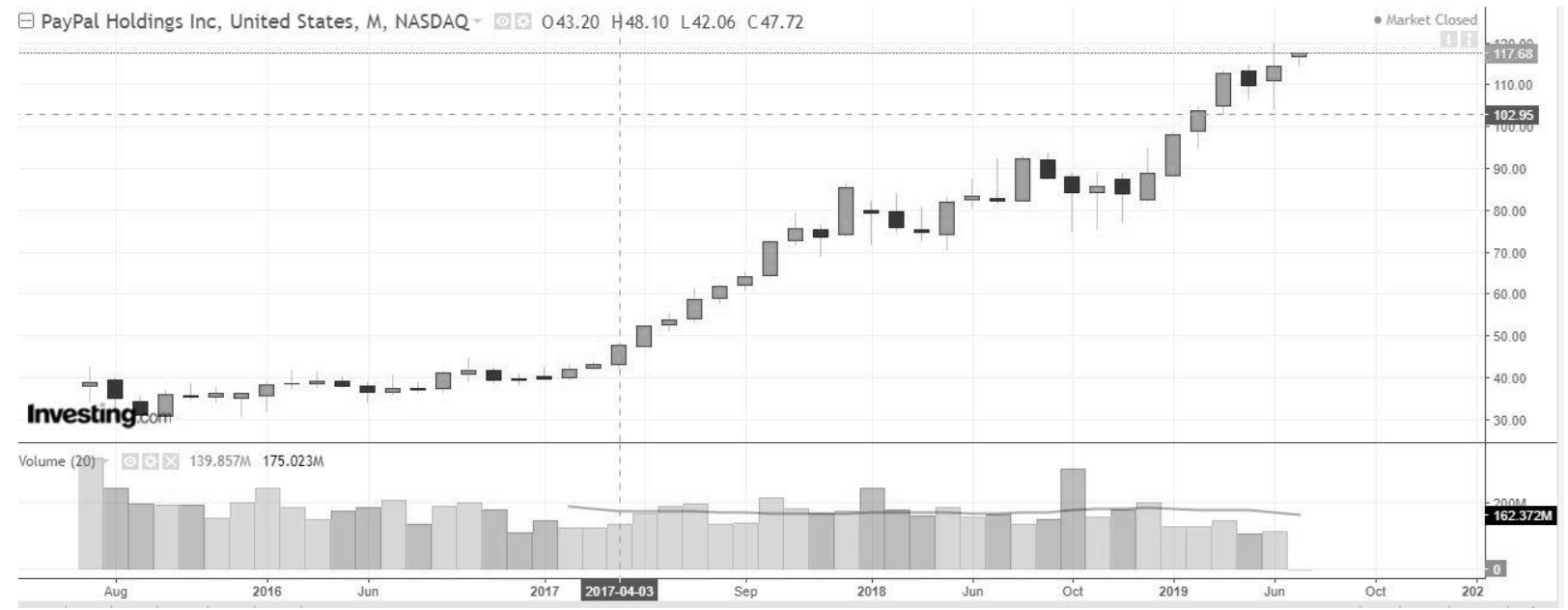

Fuente. Investing, s. f.

En la figura 3 se observa una tendencia alcista de la acción de PayPal que ha generado confianza en el mercado. Esta acción se valora sobre los 117 dólares/acción. También, de acuerdo con la figura 4, se puede identificar que las transacciones por internet de mercados minoristas aumentaron del 8,5 \% a 9,1\%. 
Figura 4. Venta comercio electrónico EE. UU.

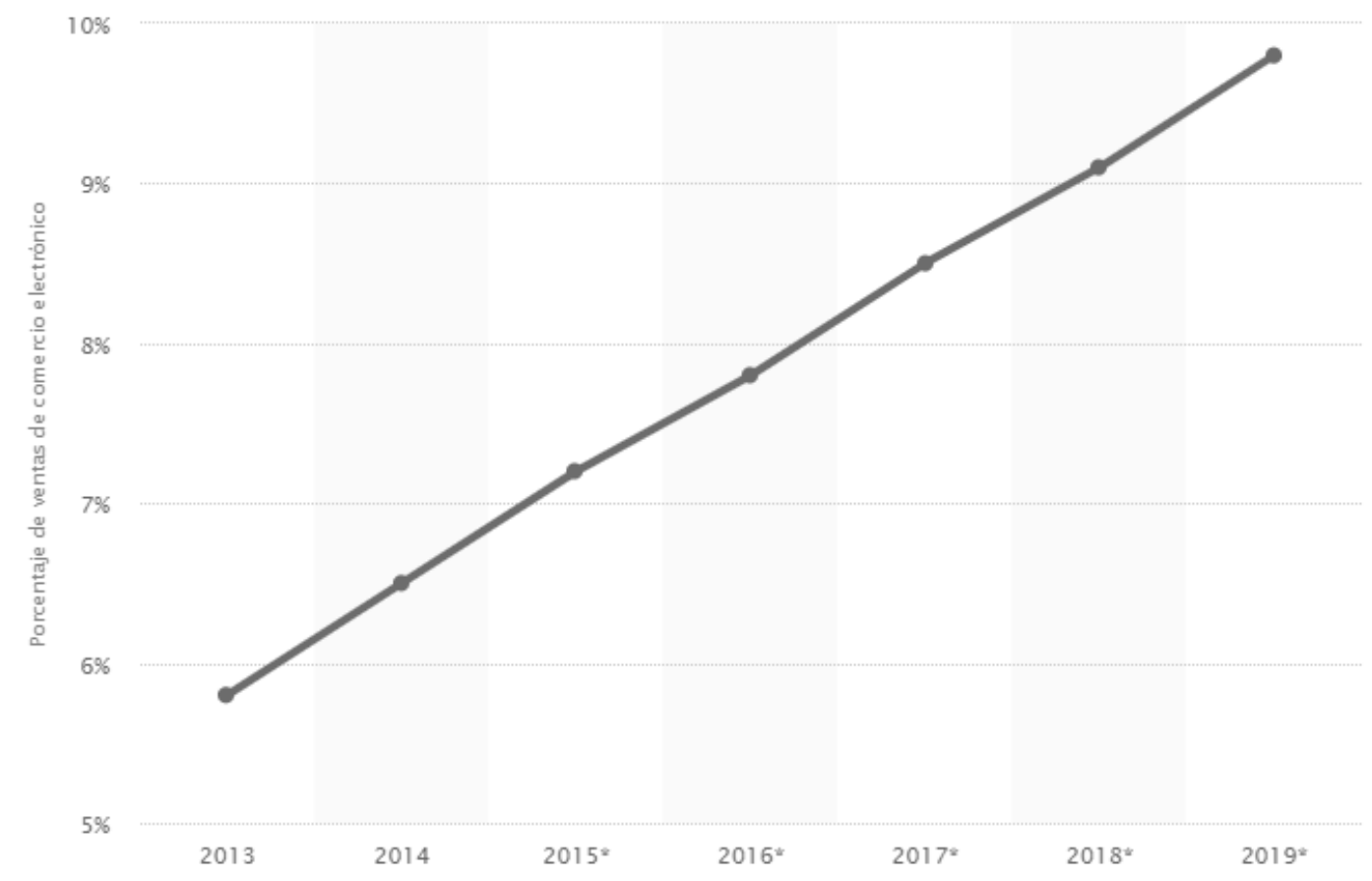

Fuente. Statista, s. f.

Según un informe del BBVA, Ant Financial es una parte fundamental de Alibaba a nivel mundial, ya que su éxito no se puede separar de la plataforma Alipay, como se conoce comercialmente la plataforma de pagos del gigante mundial chino Alibaba en comercio electrónico.

Ahora bien, en primer lugar es necesario que se expongan hitos de la historia de Alibaba. En la figura 5 se presenta una línea de tiempo sobre esta organización. 
Figura 5. Línea de tiempo Alibaba
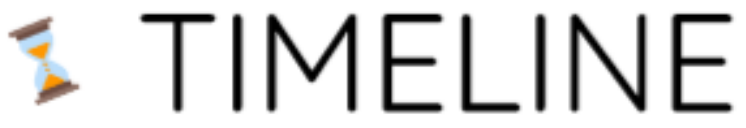

Historia de ALIBABA

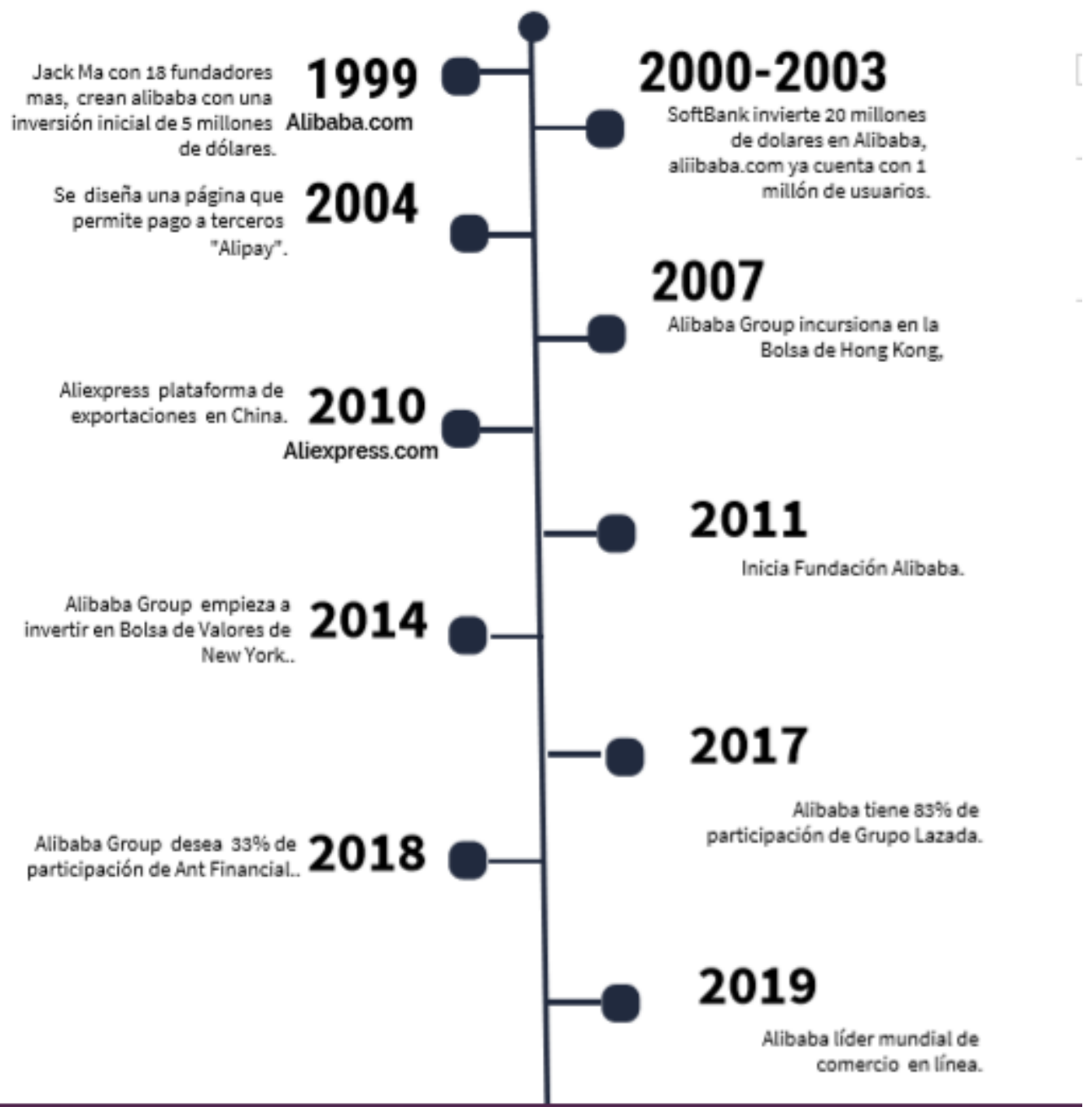

Fuente. Línea de tiempo creada en Venngage, 2019, con base en Alibaba Group, s. f.

De acuerdo con su gran trayectoria, se puede identificar que Alibaba, desde el 2014, empezó a cotizar sus acciones en la Bolsa de Valores de Nueva York con una tendencia alcista, tal como se ilustra en la figura 6. Esto ha permitido una mayor confiabilidad en el mercado y un crecimiento a lo largo de 20 años. Se ha expandido desde su origen chino hasta todos los 
países en la actualidad. La plataforma ha facilitado la conexión de productos y servicios a toda clase de clientes, entre los que se encuentran personas naturales, personas juridicas, exportadores, importadores, etc.

Figura 6. Comportamiento acción Alibaba

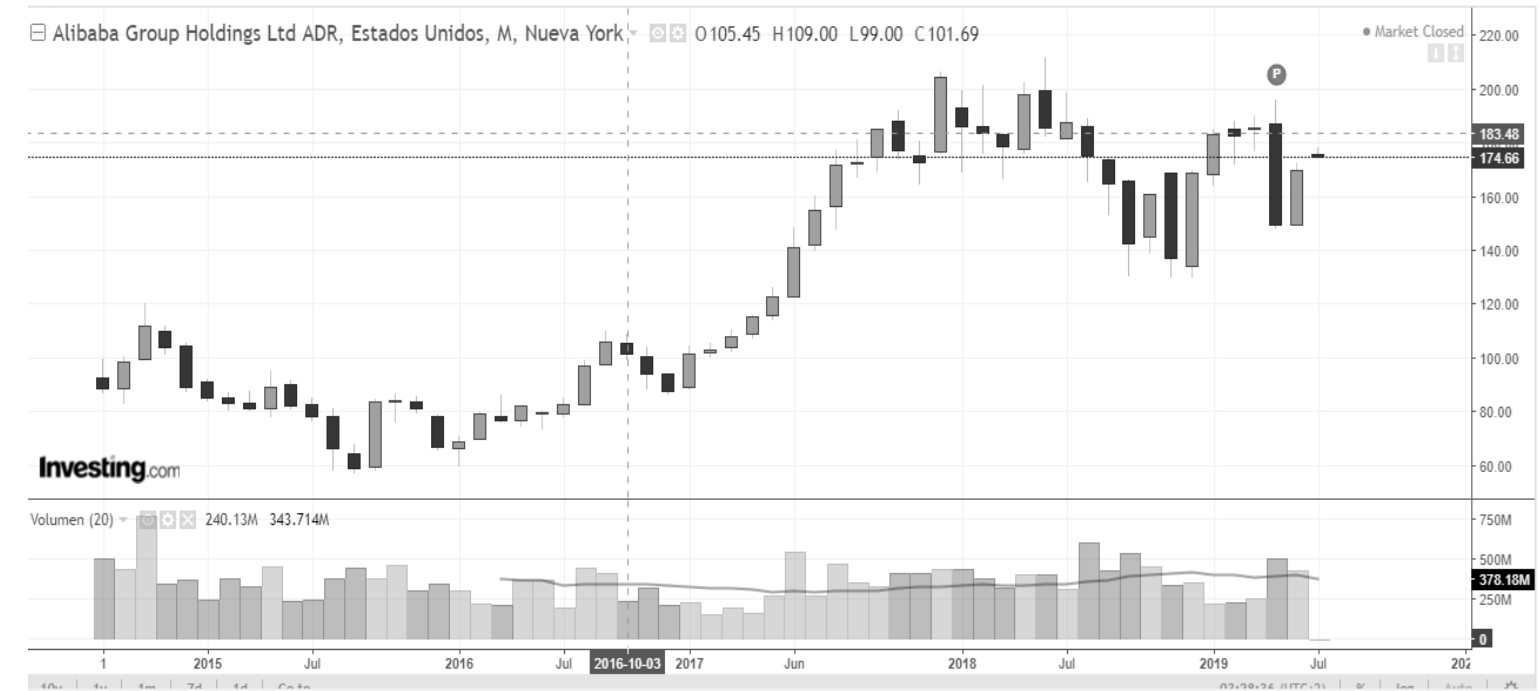

Fuente. Investing, s .f.

\section{1 ¿Por qué Ant Financial tiene que ver con Alibaba Group Holdings?}

Ant Financial, desde el punto de vista de las 12intech, es una «hormiga muy poderosa», es decir, una de la más grandes y exitosas empresas del planeta. Cuenta con una financiación de 45000 millones de dólares y su valoración se encuentra en los 60000 millones de dólares, según lo que data en el 2016.

Esta «hormiga» $\tan$ exitosa es la que administra la red de pagos Alipay de Alibaba, como antes se mostraba, «gigante comercial» en China; sus ingresos en el 2016 ascendieron a un valor de 15686 millones de dólares. Por otra parte, Ant Financial controla el 70 \% de los pagos online en China y cuenta con 100000 comercios en 70 países que utilizan su plataforma.

A diario se realizan 175 millones de transacciones por medio de Alipay con Alibaba, sobre todo de compra o venta de productos y servicios, brindando soluciones financieras. $\mathrm{Su}$ objetivo es abarcar la mayoría de mercado internacional por medio de los diferentes ramos 
del negocio fintech, razón por la cual compró en enero del 2017, por 880 millones de dólares, MoneyGram, una empresa de remesas de Estados Unidos, e invirtió en otras empresas fintech tales como India Paytm, Ascent Money — Tailandia— y Kakao Pay —Corea del Sur-.

Su éxito, en gran parte, se debe a que en Oriente la reglamentación de las fintech ha sido más flexible que en Occidente con respecto a la tecnología y la digitalización, por medio de las soluciones financieras digitales (BBVA, 2017). Así, en el 2019 Ant Financial ya vale más de 150 millones de dólares (Xie \& Steinberg, 2018).

De acuerdo con lo anterior, en el caso en el que Ant Financial (Alipay) invirtiera en el mercado de valores, se tendría en cuenta el comportamiento de PayPal, ya que esta empresa es muy similar en activos e inversiones debido a que pertenecen al mismo sector. PayPal es la pionera de pagos en América y Ant Financial lo es en Asia, dos gigantes tecnológicos creando soluciones financieras efectivas que facilitan la vida de millones de usuarios.

Con el comportamiento de la acción y su precio de ultimo día al cierre, se muestran las variaciones porcentuales en la tabla 2.

Tabla 2. Histórico acción PayPal 2015-2019

\begin{tabular}{|c|c|c|c|c|}
\hline \multicolumn{5}{|c|}{ Cierre de los precios PayPal —dólares- } \\
\hline 2015 & 2016 & 2017 & 2018 & 2019 a la fecha \\
\hline 36,2 & 39,47 & 73,62 & 84,09 & 105,3 \\
\hline & $9 \%$ & $87 \%$ & $14 \%$ & $25 \%$ \\
\hline
\end{tabular}

Fuente. Elaboración propia con datos de Investing, s. f. 
Figura 7. Variación precio acción Paypal

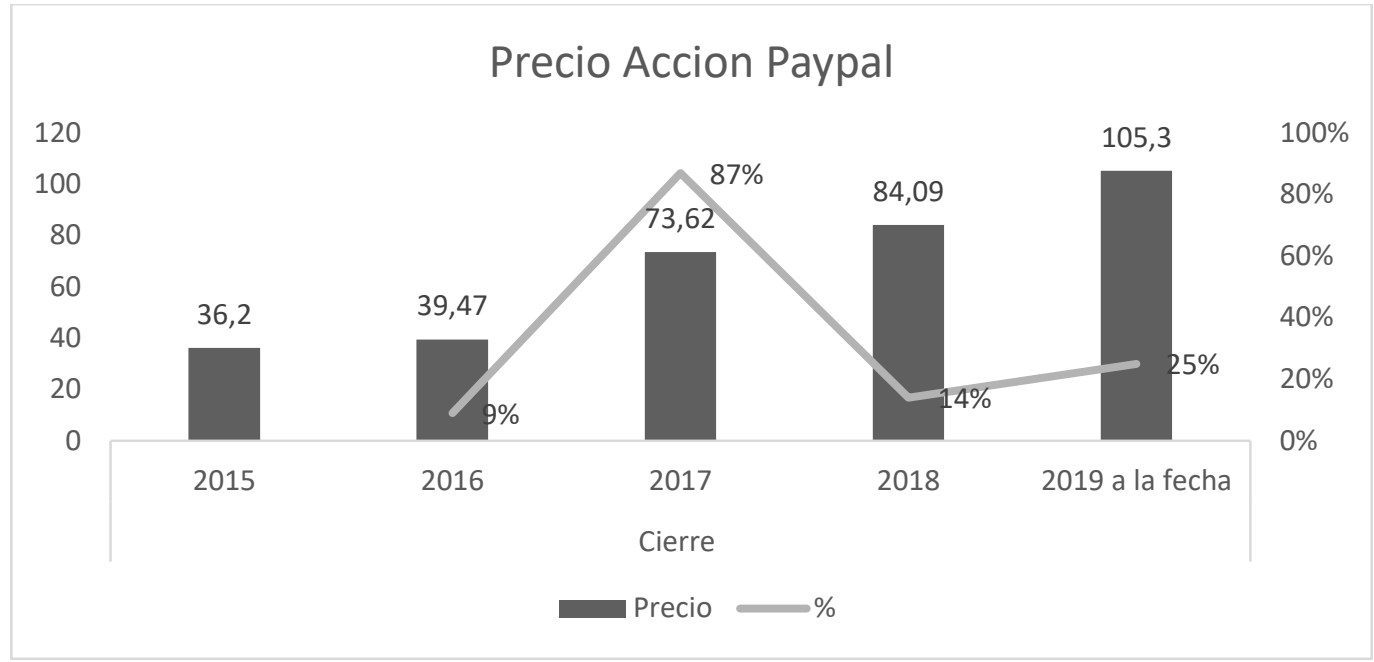

Fuente. Elaboración propia con datos de Investing, s. f.

En relación con la figura 7, la acción Ant Financial a futuro podría empezar con un precio entre 100 a 127 dólares, debido a que actualmente PayPal presenta similitud en cuanto a la actividad económica y al tipo usuarios que utilizan esta plataforma, de modo que se podría plantear una acogida importante en el mercado y el cálculo se tomaría con el valor de la empresa -150 000 millones de dólares—, y con las acciones que están circulando en el mercado en la actualidad en PayPal -1 176669 461 - (Bloomberg, 2019) se obtendría un precio aproximado de 127,47 dólares. Sin embargo, sería importante darle un valor menor, ya que - como se muestra en la tabla 2- si bien el mercado ha tenido gran acogida, dado que es una acción nueva no podría tener la misma confiabilidad que ya ha ganado PayPal.

\section{Viabilidad de inversión de las fintech en la BVC}

Antes de hablar de las fintech que podrían invertir en la BVC es importante destacar una que, aunque no es fintech, es importante y podría convertirse en una debido a su éxito en Colombia y en algunos países de Latinoamérica, tales como Brasil, Argentina, México, Perú y Chile. Se trata de Rappi, una app que ha innovado en el país, fundada en el 2015 por Simón Borrero, Sebastián Mejía y Felipe Villamil (Rappi, el nuevo unicornio de América Latina, 2018). 
Rappi es una compañía que tiene un modelo de negocio bastante particular on demand delivery, lo cual se traduce en un método en el que se despacha lo que los usuarios solicitan. Esto permite obtener un estimado de qué es lo que las personas piden en común y mejorar un estudio de mercado que abarque una mejor satisfacción al cliente. Por otra parte, a pesar de sus pérdidas por más de 56000 millones de dólares, es una entidad que busca expandir su negocio más que generar utilidades (Ojeda, 2018).

Rappi podría ser una fintech ya que, como se mencionó, es una compañía cuya tecnología ha permitido alcanzar a todo tipo de clientes y logra realizar 200000 transacciones diarias, superando todo tipo de expectativas en el exterior frente a empresas como Postmates y Doordash de Estados Unidos. En Colombia es número uno en app de domicilios y eso significa mucho para una compañía, ya que muchos de sus servicios han facilitado la vida de los colombianos, como, por ejemplo, solicitar dinero prestado y que lo entreguen en la puerta de la casa, hacer mercado por los clientes, domicilios de varios restaurantes reconocidos de la ciudad, solicitud de envío de licores o servicio de farmacia, entre otros (Miranda, 2018).

En este orden de ideas, no sería algo lejano que pudiera realizar transacciones y transferencias a entidades bancarias, cuentas de ahorros o corrientes, pago con tarjeta de crédito, como ha sucedido con su alianza con Davivienda por medio de su plataforma Daviplata, con un servicio llamado «RappiPay» que ha permitido que una alta gama de beneficios en un mismo lugar y, además, sin costos tan elevados (Monterrosa, 2019). Ahora bien, es importante resaltar que es una empresa en la que entidades como Softbank Japón- invirtieron un valor de 1000 millones de dólares, convirtiéndola en un «startup unicornio», es decir, en una compañía avaluada por más de mil millones de dólares, ubicándola en el puesto número 11 de 270 a nivel mundial en esta categoría (Miranda, 2018). Pero eso no es todo, en la ronda de recaudación de fondos en el mes de septiembre del 2018 logró que se invirtiera en ella la suma de 220 millones de dólares (Nonsoque, 2019).

En razón a lo anterior, es pertinente pensar que es una compañía que podría ser una fintech - ya que se ha expandido a lo largo de las líneas de servicio-, pues ya ha realizado 
una alianza con una entidad financiera, lo cual permite pensar que sería una fintech que brinde ese tipo de soluciones a más entidades a través de su metodología y su tecnología. Además, su posicionamiento en el mercado daría paso al conocimiento de fondo del funcionamiento de las fintech, pero no sería a corto plazo sino a largo.

A fin de proporcionar un mayor detalle de si sería posible invertir en la BVC, de acuerdo con las condiciones que esta impone para estar en capacidad de invertir y colocar acciones en el mercado, a continuación se describen tres empresas fintech: Zinobe SAS (Lineru),PayU y Conexred (Punto red), así como su posible viabilidad de invertir en el mercado colombiano.

Zinobe SAS (Lineru) no es una empresa muy conocida, pero brinda asesorías a las personas que necesitan créditos, sin filas, a través de su aplicación Lineru, la cual ha tenido éxito en nuestro país. Desde el 2012 la empresa abrió sus puertas y es una página que ha permitido a muchas personas obtener dinero sin desplazarse; ofrece un servicio de prestamos $100 \%$ online que facilita el proceso, tarda tan solo 15 minutos y al día siguiente hábil entrega el dinero (Lineru, 2019).

Con respecto a la inversión que podría realizar en la BVC, según los requisitos de esta, se puede determinar por sus indicadores más relevantes que ha presentado un crecimiento en sus ventas en el 2017 de 1,8 millones de dólares y en el 2018 de 4,2 millones de dólares, más del 100 \% en lo que respecta del 2017 al 2018; además sus utilidades netas han estado por encima del $200 \%$ en la diferencia del 2017 -USD 615 319- a 2018 -USD 1,9 millones-. Sin embargo, sus deudas abarcan 124 puntos porcentuales - proporcional a sus ganancias- y su patrimonio abarca 2,1 millones de dólares, mas a la luz de invertir en la bolsa colombiana se descartaría esta opción, ya que su patrimonio es inferior y representa tan solo el $97 \%$ de lo que solicitan. No obstante, debe tenerse en cuenta que su patrimonio aumentó un 179 \% en el 2018 y eso sería un buen comienzo para que se aumente su capital, teniendo en cuenta, también que lleva más de cinco años en el mercado y ha mantenido su objeto social. 
En lo que corresponde al 2016 tuvo pérdidas por un valor de USD 161 809, mientras en los años 2017 y 2018 la compañía ha producido ganancias, motivo por el cual en el momento no estaría cumpliendo con las exigencias mínimas que solicita la bolsa de valores (EMIS University [Bechmark], s. f.)

En segundo lugar se encuentra la empresa PayU Colombia. Es una entidad famosa porque ha provisto a muchas compañías sus servicios a través de plataformas de pagos. Sus inicios datan del 2002, cuando un economista, José Fernando Vélez, y Martín Schrimpff, un ingeniero mecánico, pensaron en una solución que facilitara las compras y las ventas de productos y servicios. Era algo arriesgado para aquel entonces, ya que la tecnología en Colombia no estaba en auge. Para el 2010 recibieron el reconocimiento Emprendedores Endeavor, y en el 2011 se unieron a Naspers, quienes manejaban la red PayU Group que se establecía en Europa, Asia y África. En la actualidad se llaman PayU Latam, la más grande red de pagos en Latinoamérica y mundialmente amparada por PayU Group (Colombia.com 2013).

Esta compañía maneja varios métodos de pago, se ha asociado con entidades como Gtech - Baloto - o Efecty a fin de generar un ingreso más ameno a personas que no confían mucho en las transacciones por internet.

De acuerdo con estos factores, se analiza si sería viable que cotizara en la BVC a la luz de sus requisitos. Su patrimonio actual corresponde a 1,3 millones de dólares, lo que representa el $64 \%$. En los últimos 15 años ha realizado su actividad económica principal de proveedor de internet y servicios financieros a empresas y personas naturales. En lo que respecta a su utilidad operacional se pueden identificar unas pérdidas que ascienden a 3,7 millones de dólares en el último año —aumento de 2017 a 2018 en un 142 \%—, aunque sus ingresos por ventas aumentaron un 11,67 \% y sus deudas en un 58,4\%. Por otra parte, de acuerdo con un artículo de Dinero, la empresa PayU ha logrado posicionarse en el mercado, crecer en un $40 \%$ y abarcar un mercado de 200000 comercios y alrededor de 10 millones de compradores. Su mayor desafío desde el 2018 ha sido el de «PayU te fía», con el que ha 
incursionado en el mercado facilitándole a las personas que lo utilicen la posibilidad de comprar algo y no pagarlo de manera inmediata (Fiando, PayU quiere hacer crecer, 2018).

Es necesario que la empresa evalúe la forma en que se está endeudando para, así mismo, lograr un equilibrio y estar en capacidad de crecer más de lo que lo ha hecho en los últimos cinco años.

Finalmente, Conexred - Punto Red — es una empresa fundada por Andrés Albán. En un principio era una empresa única y exclusivamente de recargas y solo estaba con tres operadores de servicios celulares. En el 2007 se crea una red administrativa que permitía recargas a través de un punto de red. En la actualidad maneja todo de tipo de transacciones, como, por ejemplo, envío de remesas o giros nacionales. Además, tiene convenio con varios bancos a nivel nacional, lo cual realiza través de negocios - ya sean pequeños o grandesbrindándole servicios tecnológicos con plataformas que permiten facilitar los procesos de pagos (Punto Red, s. f.).

En conformidad con los requerimientos de la BVC se puede evidenciar que la empresa cuenta con un patrimonio de 4,1 millones de dólares, lo que representa un 191 \% por encima de los 7000 millones que solicita la bolsa colombiana; se ha mantenido en su actividad económica, entre otras, las telecomunicaciones, y en lo que respecta a su utilidad operacional ha obtenido ganancias en el 2015 de 3,2 millones de dólares, en el 2016 de 2,2 millones de dólares, en el 2017 de 1,5 millones de dólares y en el 2018 por un valor de 960955 dólares - con respecto al año anterior disminuyó en $37 \%$ - En lo que corresponde a sus deudas, aumentaron en un 1,46\% en lo que se refiere al periodo 2017-2018. Es una empresa que podría apalancar algunas de esas deudas con emisión de acciones, ya que cumple la mayoría de puntos que solicita la bolsa de valores para invertir (EMIS University [Bechmark], s. f.).

Por otra parte, Colombia Fintech ha logrado grandes avances, si se tiene en cuenta que en el 2019 la BVC se ha aliado con ellos para alcanzar una mayor digitalización en el mercado de capitales. Así, próximamente lanzará al mercado de las pymes una plataforma que realizará junto con una fintech, la cual se llamará A2censo, con la que busca que los 
emprendedores puedan emitir bonos de deuda y que cualquier persona del país pueda invertir en ellos sin restricción (Loaiza, 2019).

\subsection{Aproximación precio acciones fintech en la BVC}

Con el comportamiento del índice Nasdaq con respecto al de la acción PayPal, en la figura 8 se puede identificar que la acción ha subido en paralelo con el índice, a pesar de su pequeña participación. Así se puede observar en el precio de la acción con respecto a lo equivale el índice, pues su movimiento en el mercado ha tenido una correlación directa en el 2016 y la acción creció un 9 \% mientras que el índice creció un 6 \%, lo que quiere decir que la acción apalancó el crecimiento del índice. Sin embargo, en el 2018 se ha visto una diminución tanto en PayPal como en Nasdaq, pero de nuevo se ha recuperado a septiembre del 2019. Con este referente podríamos dar una estimación de lo que podría ser el precio de la acción, así como al adaptar la situación en el mercado colombiano con el índice del Colcap, que no maneja el mismo volumen de la bolsa americana, dada la dimensión de la economía tercermundista que maneja el país.

Figura 8. Comparativo índice Nasdaq con acción Paypal

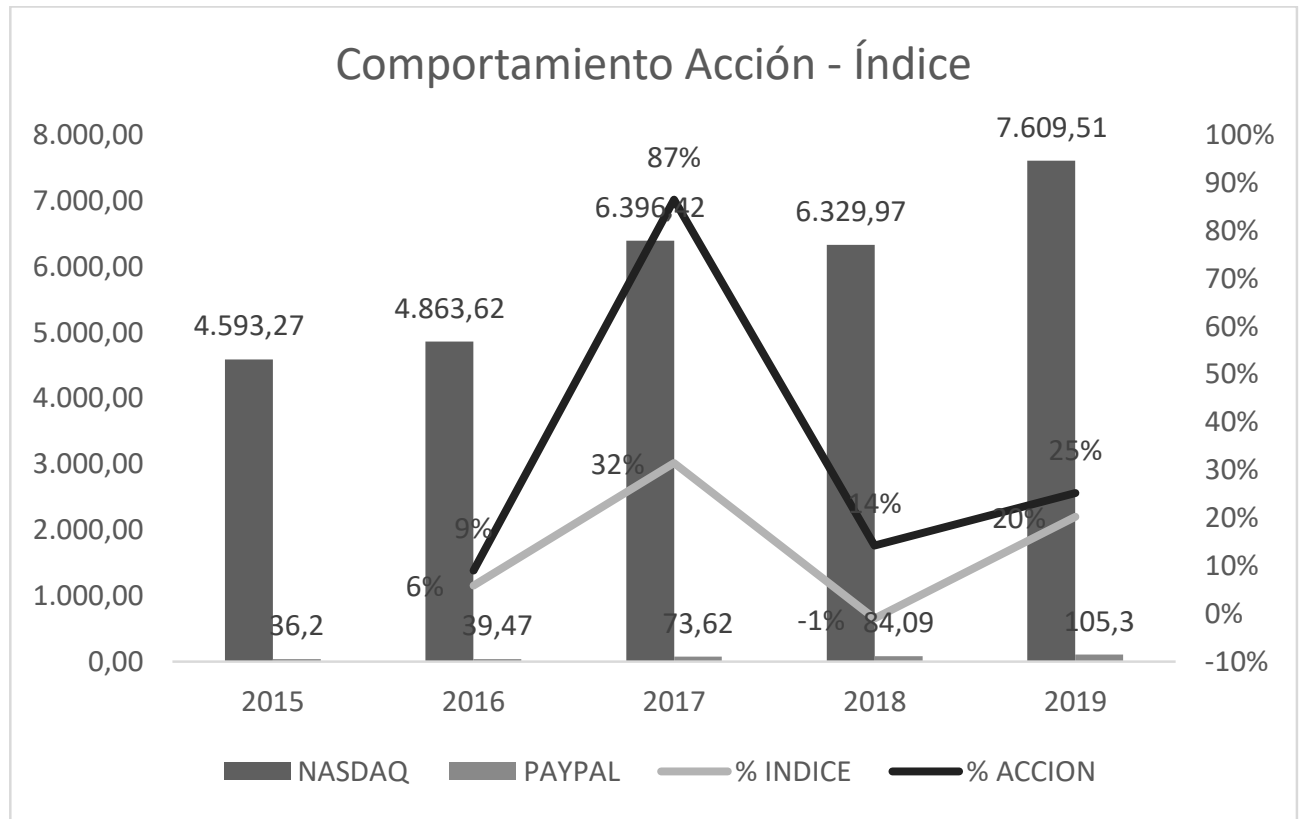

Fuente. Elaboración propia con base en datos de Investing, s. f. 
Dado el comportamiento que ha tenido el Colcap en los últimos cinco años, se daría una estimación aproximada del precio de una acción fintech en el mercado colombiano. A esto es necesario añadirle que el índice Colcap, en comparación con el Nasdaq que se muestra en la gráfica de la figura 8, ha tenido variaciones similares a los de la gráfica de la figura 9, ya que Colombia está relacionada intrínsicamente con la situación de este, debido a que Estados Unidos es potencia mundial y lo que sucede allí afecta el mercado.

Con esta estimación del comportamiento de la acción se puede apreciar que la acción en Colombia podría tomar fuerza. El fenómeno fintech ha crecido en los últimos dos años y por tal motivo sería de gran confiabilidad a la hora de realizar inversiones. Se podría decir que al compararlo con el mercado americano se puede identificar que si la acción estuviera en circulación ya habría crecido en un25 \% y ayudaría al índice a aumentar su valor.

Figura 9. Variación índice Colcap

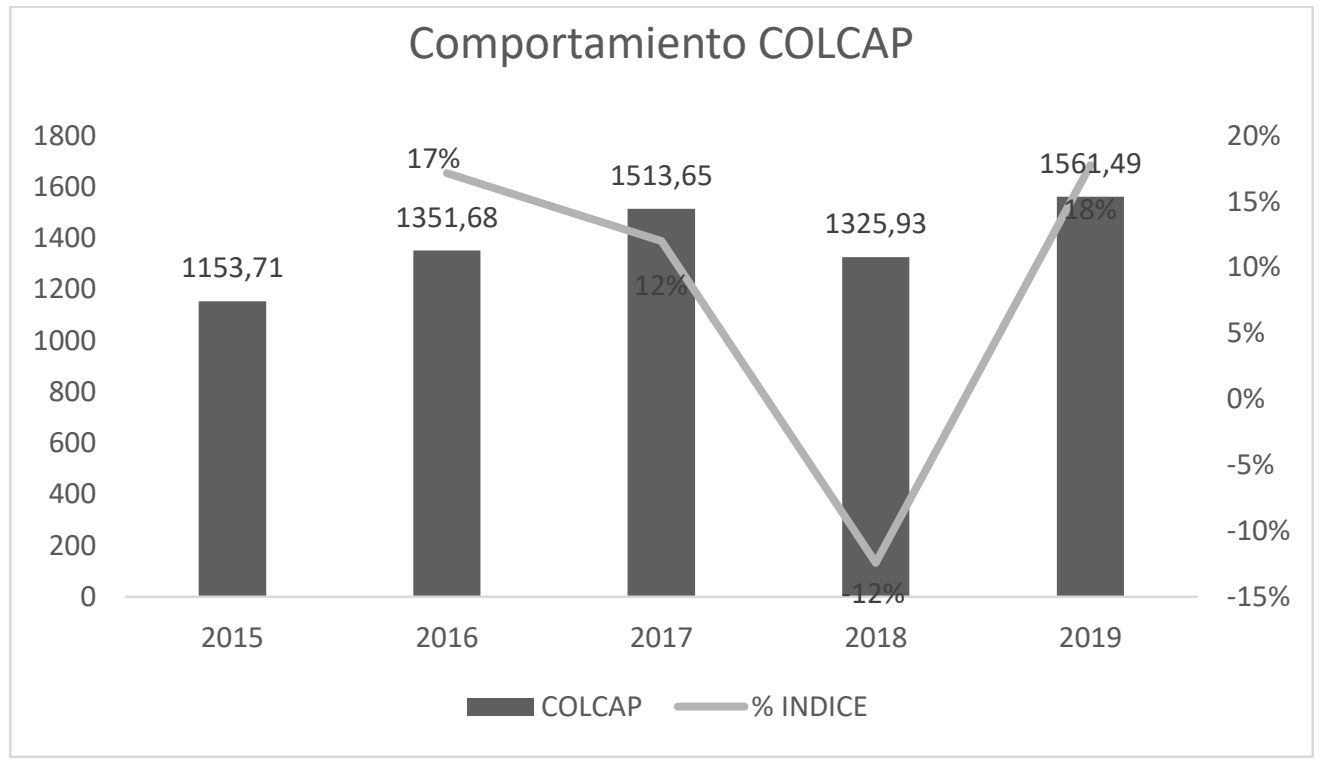

Fuente. Elaboración propia con datos de Bloomberg, s. f. 


\section{Conclusiones}

Después de esta investigación se logra identificar que las fintech en Colombia pueden llegar a ser un potencial en cuanto a inversión en la bolsa de valores, sin embargo, es importante tener en cuenta que no todas las empresas podrían invertir debido a las solicitudes que la entidad hace. Por esta razón, sería indispensable que la BVC replantee las condiciones para invertir y establezca unas más asequibles a las empresas que permitan mayor flexibilidad y accesibilidad.

A partir de la investigación y la metodología utilizada se puede concluir que la BVC aún no está lista para invertir y posicionar acciones con las fintech, debido a la situación de la economía actual, en la cual las empresas quiebran fácilmente ya sea, por el alto nivel tributario que manejan; con el PIB en el 3,0 \% (DANE, s. f.), lo que quiere decir que los precios de los productos y servicios incrementaron; por otro factor externo como el dólar —que está por encima de los 3400 pesos (Set-icap, s. f.) - , lo cual se traduce en un déficit dentro del país; o bien por la situación de Estados Unidos, la guerra comercial que mantiene con China y sus altos aranceles para el transporte de los productos. Por esto, aunque la BVC no es una entidad pública y su función es administrar los procedimientos de negociación a través de un sistema, podría transformarse ante esta problemática que no se presenta solo con acciones fintech, sino en general con las demás acciones que invierten pero que no cuentan con volúmenes tan significativos; muchas veces el índice Colcap ha presentado caídas importantes, a tal punto que los expertos recomiendan que las empresas importantes como Alpina, Bavaria o Empresas Públicas de Medellín (EPM) inviertan a fin de empujar al alza del índice (González, 2018).

Con la metodología utilizada se trató de presentar un análisis objetivo que permita visualizar un nicho de negocios que se encuentra en auge en el mundo, y que también en Colombia puede ser parte de todos los sectores, debido a su éxito y a lo que ha logrado en los dos años que lleva Colombia Fintech innovando y realizando los correspondientes procesos para que estas entidades se logren destacar en el territorio y lleguen a todos los lugares del país. En este orden de ideas, podría existir la posibilidad de que a futuro sea el 
apalancamiento de la economía del país, como lo ha sido Alibaba en China, que ha extendido su modelo de negocio, creando un holding que permite abarcar ampliamente las necesidades de las personas que manejan su información y desean comprar productos y servicios a través de celulares, computadores, etc. Además, la BVC ha avanzado al aliarse con Colombia Fintech y entidades del Estado como la Superfinanciera, la cual ha creado el grupo de trabajo InnovaSFC como parte del programa de inclusión financiera. Sin embargo, aún falta mucho por realizar. Así, por ejemplo, se requiere una ley o norma que establezca el debido proceso del mundo digital, dado que esto se ha realizado a nivel mundial por medio de las buenas prácticas. Otra cosa que se debe considerar como un ejemplo es la forma como el Medio Oriente ha dado cierta flexibilidad a estos negocios, cosa que no ha pasado en países como Estados Unidos en donde han tenido ciertas restricciones con respecto al uso de los medios; a pesar de lo que se ha logrado, también le falta a esta potencia generar facilidad y permitir así un mejor manejo de los recursos.

$\mathrm{Al}$ profundizar en los gráficos de algunas fintech se puede evidenciar que Colombia necesita mayor preparación en cuanto al manejo de estas organizaciones, ya que es un procedimiento innovador que ha llegado a muchas personas. En este sentido, es preciso resaltar cómo, a pesar del progreso que se ha tenido en cuanto a avance tecnológico, aún falta mucho en educación financiera, específicamente sobre la forma en que se manejan las plataformas, ampliar el conocimiento sobre las facilidades que brindan y lo útiles que pueden llegar a ser. Si bien existen programas que han implementado las entidades financieras y han enseñado a las personas acerca de un mayor aprovechamiento de los productos financieros - por ejemplo, un mejor ahorro, no sobre endeudarse, etc.-, con respecto a inversión en bolsa la BVC ha implementado ciertos programas y cursos que ayudan a instruir acerca de lo que se debe y no se debe hacer. Además, ha contado con el apoyo de la Superintendencia Financiera con su grupo de trabajo InnovaSFC, así como de Asobancaria, lo cual también se ha interpuesto con los bancos, las cooperativas y las aseguradoras. No obstante, falta mucho conocimiento y preparación por parte de las personas mayores de 40 años, ya que desconfían del correcto funcionamiento y muchas veces prefieren pagar un recibo de forma presencial 
arealizar un pago virtual por medio de las plataformas digitales de los bancos, bien sea PSE o bien PayU, entre otras.

\section{Referencias}

Alibaba Group. (s. f.). History and milestones. Recuperado de https://bit.ly/31XZHNR

Asobancaria. (2018). Segmento fintech en Colombia: ¿en qué vamos? Semana Económica 1162. Recuperado de https://bit.ly/209E9pe

Avendaño, A. (24 de marzo de 2016). ¿Qué pasaría si Donald Trump fuera presidente de Estados Unidos? Washington Post. Recuperado de https://wapo.st/3gCSFII

Barrera, A. (2019). ¿Qué es Paypal y cuál es su historia de éxito? Next U. Recuperado de https://bit.ly/3iIHUAe

BBVA. (13 de marzo de 2017). Así es Ant Financial, la mayor «fintech» del planeta. Recuperado de https://bbva.info/20406Gi

Bloomberg. (s. f.). Paypal. Recuperado de https://bloom.bg/3iLiOAv

Bolsa de Valores Colombia (BVC). (2018). Ser emisor BVC, una decisión estratégica. Recuperado de https://bit.ly/3iLiPo3

Bolsa de Valores Colombia (BVC). (s. f.). Mercado de renta variable. Recuperado de https://bit.ly/3edulVM

Cámara de Comercio de Bogotá. (2018). Un breve recorrido por la historia del fintech. Recuperado de https://bit.ly/2Z8LVWN

Cañete, I. (25 de abril de 2018). ¿Qué es una «startup»? BBVA. Recuperado de https://bbva.info/38ECNMC

Colombia.com (22 de agosto de 2013). Pagosonline ahora es PayU Latam. Recuperado de https://bit.ly/2ZTge2K

Colombia Fintech. (2018). Acerca de Colombia Fintech. Recuperado de https://bit.ly/3eegLlb

Colombia Fintech. (s. f.). Nuestra historia desde el 2017. Recuperado de https://bit.ly/208PqXa

DANE. (s. f.). Cuentas nacionales. Recuperado de https://bit.ly/2ZSLQWm

Decreto 1491 de 2015. (13 de julio de 2015). Por el cual se modifica el Decreto número 2555 de 2010 en lo relacionado con la reglamentación aplicable a las Sociedades Especializadas en Depósitos y Pagos Electrónicos, (SEDPE) y se dictan otras disposiciones. Diario Oficial, núm. 49572. Ministerio de Hacienda y Crédito Público. 
Decreto 1357 de 2018. (31 de julio de 2018). Por el cual se modifica el Decreto 2555 de 2010 en lo relacionado con la actividad de financiación colaborativa. Diario Oficial, núm. 50.671. Ministerio de Hacienda y Crédito Público.

EMIS University (Bechmark). (s. f.). Estados financieros.

Fiando, PayU quiere hacer crecer el comercio electrónico en Colombia. (26 de abril de 2018). Dinero. Recuperado de https://bit.ly/2DnYUvs

González, C. E. (6 de marzo de 2018). Las empresas que los inversionistas quieren ver en la Bolsa de Valores de Colombia. La República. Recuperado de https://bit.ly/2W285bc

Investing. (s. f.). PayPal Holdings Inc (PYPL). Recuperado de https://bit.ly/2ZbsooT

Investing. (s.f.). Alibaba Group Holdings Ltd ADR (BABA). Recuperado el 2019, de https://bit.ly/3fbPD7z

Investing. (s. f.). Índices del mundo. Recuperado de https://bit.ly/38GhlqL

Ley 1735 de 2014. (21 de octubre de 2014.) Por la cual se dictan medidas tendientes a promover el acceso a los servicios financieros transaccionales y se dictan otras disposiciones. Diario Oficial, núm. 49311, Ministerio de Hacienda y Crédito Público.

Lineru. (s. f.). Quiénes somos. Recuperado de https://bit.ly/2VZexzT

Loaiza, A. V. (8 de Julio de 2019). La Bolsa de Valores de Colombia es nuevo miembro de la asociación Colombia Fintech. La República. Recuperado de https://bit.ly/3iFTImK

Los momentos destacados de la toma de posesión de Donald Trump como el $45^{\circ}$ presidente de Estados Unidos. (21 de enero de 2017). BBC Mundo. Recuperado de https://bbc.in/3iJowDi

Miranda, B. (26 de octubre de 2018). Rappi, el «Amazon de Colombia» que se convirtió en el emprendimiento más exitoso del país - y que genera protestas en algunas ciudades de América Latina-. BBC Mundo. Recuperado de https://bbc.in/38DrOTO

Molina, D. I. (2016). Fintech: lo que la tecnología hace por las finanzas. Barcelona: Profit.

Monterrosa, H. (22 de mayo de 2019). Alianza entre Davivienda y Rappi permitirá transacciones sin costos para sus usuarios. La República. Recuperado de https://bit.ly/31VLf96

Nonsoque, J. C. (29 de abril de 2019). SoftBank invertirá cerca de US\$1.000 millones en la plataforma de domicilios Rappi. La República. Recuperado de https://bit.ly/2VZGs2N

Ojeda, D. (9 de septiembre de 2018). ¿Por qué Rappi vale US\$1.000 millones si genera pérdidas? El Espectador. Recuperado de https://bit.ly/3efSnzA

Punto Red. (s. f.). Nuestra historia. Recuperado de https://bit.ly/2CfreQ0 
Rappi, el nuevo unicornio de América Latina, busca llegar a nuevos territorios en 2019. (14 de octubre de 2018). La República. Recuperado de https://bit.ly/209JyNi

Set-icap. (s. f.). Información del dólar. Recuperado de https://bit.ly/2W1co60

Statista. (s. f.). Estados Unidos: porcentaje de comercio electrónico en ventas minoristas 2013-2019. Recuperado de https://bit.ly/3iJANHF

Tras acuerdo con Rappi, Davivienda entra a ser parte de Rappipay. (22 de mayo de 2019). Dinero. Recuperado de https://bit.ly/2VXy0kA

Xie, S. Y.; Steinberg, J. (7 de junio de 2018). Jack Ma's Ant Financial valued around $\$ 150$ billion after funding round. The Wall Street Journal. Recuperado de https://on.wsj.com/32aK1qP 